\title{
Functional histology of the skin in the subterranean African giant mole-rat: Thermal windows are determined solely by pelage characteristics
}

\author{
Lucie Pleštilová ${ }^{1}$, Jan Okrouhlík ${ }^{1}$, Hynek Burda ${ }^{2}$, Hana Sehadova ${ }^{3,4}{ }^{\text {, Eva Valesky }}{ }^{5}$, Radim Šumbera ${ }^{\text {Corresp. } 1}$ \\ ${ }^{1}$ Department of Zoology, Faculty of Science, University of South Bohemia, České Budějovice, Czech Republic \\ 2 Department of Game Management and Wildlife Biology, Faculty of Forestry and Wood Sciences, Czech University of Life Sciences, Prague, Czech \\ Republic \\ 3 Department of Molecular Biology and Genetics, Faculty of Science, University of South Bohemia, České Budějovice, Czech Republic \\ 4 Institute of Entomology, Biology Centre of the Czech Academy of Sciences, České Budějovice, Czech Republic \\ 5 Department of Dermatology, Venereology and Allergology, University Hospital, Johann Wolfgang Goethe Universität Frankfurt am Main, Frankfurt am \\ Main, Germany \\ Corresponding Author: Radim Šumbera \\ Email address: sumbera@prf.jcu.cz
}

Excavation of burrows is an extremely physically demanding activity producing a large amount of metabolic heat. Dissipation of its surplus is crucial to avoid the risk of overheating, but in subterranean mammals, it is complicated due to the absence of notable body extremities and high humidity in their burrows. Using IR-thermography in a previous study on two species of African mole-rats revealed that body heat is dissipated mainly through the ventral body part, which is notably less furred. Here, we analyzed the dorsal and ventral skin morphology, to test if dermal characteristics could contribute to higher heat dissipation through the ventral body part. The hickness of the epidermis and dermis and the presence, extent, and connectivity of fat tissue in the dermis were examined using routine histological methods, while vascular density was evaluated using fluorescent dye and confocal microscopy in the giant mole-rat Fukomys mechowii. Like in other hitherto studied subterranean mammals, no subcutaneous adipose tissue was found. All examined skin characteristics were very similar for both dorsal and ventral regions: relative content of adipose tissue in the dermis (14.4 $\pm 3.7 \%$ dorsally and $11.0 \pm 4.0 \%$ ventrally), connectivity of dermal fat $(98.5 \pm 2.8 \%$ and $95.5 \pm 6.8 \%)$, vascular density (26.5 $\pm 3.3 \%$ and $22.7 \pm 2.3 \%$ ). Absence of large differences in measured characteristics between particular body regions indicates that the thermal windows are determined mainly by the pelage characteristics. 


\section{Functional histology of the skin in the subterranean}

2 African giant mole-rat: Thermal windows are

\section{3 determined solely by pelage characteristics}

6 Lucie Pleštilová1, Jan Okrouhlík ${ }^{1}$, Hynek Burda ${ }^{2}$, Hana Sehadová $^{3,4}$, Eva Maria Valesky ${ }^{5}$, Radim

7 Šumbera ${ }^{1}$

$9{ }^{1}$ Department of Zoology, Faculty of Science, University of South Bohemia, České Budějovice, 10 Czech Republic

112 Department of Game Management and Wildlife Biology, Faculty of Forestry and Wood

12 Sciences, Czech University of Life Sciences, Prague, Czech Republic

$13{ }^{3}$ Department of Molecular Biology and Genetics, Faculty of Science, University of South

14 Bohemia, České Budějovice, Czech Republic

$15{ }^{4}$ Institute of Entomology, Biology Centre of the Czech Academy of Sciences, České Budějovice, 16 Czech Republic

$17{ }^{5}$ Department of Dermatology, Venereology and Allergology, University Hospital, Johann

18 Wolfgang Goethe Universität Frankfurt am Main, Germany

20 Corresponding Author:

21 Radim Š́umbera ${ }^{1}$

22 Branišovská 1760, České Budějovice, 37005, Czech Republic 
23 Email address: sumbera@prf.jcu.cz

\section{Abstract}

25 Excavation of burrows is an extremely physically demanding activity producing a large amount 26 of metabolic heat. Dissipation of its surplus is crucial to avoid the risk of overheating, but in 27 subterranean mammals; it is complicated due to the absence of notable body extremities and high 28 humidity in their burrows. Using IR-thermography in a previous study on two species of African 29 mole-rats revealed that body heat is dissipated mainly through the ventral body part, which is notably less furred. Here, we analyzed the dorsal and ventral skin morphology, to test if dermal characteristics could contribute to higher heat dissipation through the ventral body part. The hickness of the epidermis and dermis and the presence, extent, and connectivity of fat tissue in the dermis were examined using routine histological methods, while vascular density was evaluated using fluorescent dye and confocal microscopy in the giant mole-rat Fukomys mechowii. Like in other hitherto studied subterranean mammals, no subcutaneous adipose tissue was found. All examined skin characteristics were very similar for both dorsal and ventral regions: relative content of adipose tissue in the dermis $(14.4 \pm 3.7 \%$ dorsally and $11.0 \pm 4.0 \%$ ventrally), connectivity of dermal fat $(98.5 \pm 2.8 \%$ and $95.5 \pm 6.8 \%)$, vascular density $(26.5 \pm 3.3$ $\%$ and $22.7 \pm 2.3 \%$ ). Absence of large differences in measured characteristics between particular body regions indicates that the thermal windows are determined mainly by the pelage

41 characteristics.

\section{Introduction}

44 Mammals are able to maintain a stable and relatively high body temperature in a wide range of 45 ambient temperatures, which is achieved by heat production and heat loss regulation ( $\mathrm{McNab}$, 
46 2002; Withers et al., 2016). When mammals perform energy consuming activities, production of

47 body heat increases substantially, yet surplus heat would cause overheating and has to be

48 dissipated (McNab, 2002; Schmidt-Nielsen, 1997; Sherwood, Klandorf \& Yancey, 2013).

49 Mammals can lose heat by physical routes i.e. radiation, convection, conduction, or evaporation.

50 Evaporation is the most effective way of cooling, however, it is limited in water-saturated

51 environments or when water for sweating is not available (Baldo, Antenucci \& Luna, 2015;

52 McNab, 2002; Withers et al., 2016).

53 Heat dissipation in mammals can be enhanced in body areas known as thermal windows

54 (Feldhamer et al., 2015; Withers et al., 2016). These areas are usually sparsely haired and

55 situated at body appendages as pinnae in elephants or rabbits (Mohler \& Heath, 1988;

56 Weissenböck et al., 2010), tail in coypus and beavers (Krattenmacher \& Rübsamen, 1987; Steen

$57 \&$ Steen, 1965), or feet in foxes or otters (Klir \& Heath, 1994; Kuhn \& Meyer, 2009). Thermal

58 windows are usually well vascularized with numerous arteriovenous anastomoses, regulating the

59 heat transfer by vasodilatation and vasoconstriction (Bryden \& Molyneux, 1978; Khamas et al.,

60 2012; Vanhoutte et al., 2002). There are, for example, two main blood vessel plexus beneath the

61 dark patches of giraffe skin considered as thermal windows, which facilitates heat exchange with

62 the environment (Ackermann, 1976; Mitchell \& Skinner, 2004). The blood vessel walls are also

63 thinner in the patches than in non-patch lighter skin (Mitchell \& Skinner, 2004). In the proximal

64 region of the wing of the Brazilian free-tailed bat (Tadarida brasiliensis), a network of arteries

65 and veins positioned perpendicular to the body has been found, which is unique among bats and

66 which is considered to be a thermal window allowing effective thermoregulation during

67 migration (Reichard et al., 2010). 
Due to its low heat conductivity, a fat layer can be an important component of heat

69

70

71

72

73

74

75

76

77

78

79

80

81

82

83

84

85

86

87

88

89

conservation, particularly for aquatic mammals living in cold water (Bryden, 1964; Kvadsheim

\& Folkow, 1997; Liwanag et al., 2012). These mammals have a thick continuous insulative

subcutaneous fat layer all over the body, except for the extremities used for active

thermoregulation (Khamas et al., 2012; Schmidt-Nielsen, 1997). The insulative properties of fat layer have also been proven in much smaller laboratory mice (Kasza et al., 2014, 2016).

Heat dissipation is particularly challenging in burrowing mammals. Digging in a

mechanically resistant substrate is energetically demanding as it produces a lot of metabolic heat (e.g. Ebensperger \& Bozinovic, 2000; Lovegrove, 1989; Luna \& Antenucci, 2007; Zelová et al., 2010). We estimate that subterranean mammals (i.e. burrowing mammals, which forage underground) spend several hours per day by digging new burrows and transporting excavated soil. Such activity inevitably produces a surplus of metabolic heat. However, the burrow atmosphere is typically very humid (with relative humidity often exceeding $90 \%$ ) (reviewed in Burda, Šumbera \& Begall, 2007), which impairs the efficacy of evaporative cooling (Šumbera, 2019). Moreover, subterranean mammals usually lack longer body appendages, which would facilitate heat radiation (see above). The ability to lose heat via convection in sealed tunnels with very restricted (if any) air currents is also extremely limited (Baldo, Antenucci \& Luna, 2015; Burda, Šumbera \& Begall, 2007). The best way to dissipate a surplus of heat seems to be cooling via conduction through appressing the body surface to the colder tunnel walls. Indeed, relatively high thermal conductance in subterranean rodents suggests this way of cooling (Buffenstein, 2000).

It was speculated that in subterranean mammals, the ventral body surface is relevant for heat dissipation as indicated by its shorter and less dense fur (Cutrera \& Antenucci, 2004;

Peer] reviewing PDF | (2019:11:43267:1:2:NEW 2 Mar 2020) 
91 Šumbera et al., 2007). In two species of African mole-rats (Bathyergidae), the silvery mole-rat

92 Heliophobius argenteocinereus and the giant mole-rat Fukomys mechowii, the importance of the

93 less furred ventral body part as the main thermal window was supported also by infrared

94 thermography (Šumbera et al., 2007; Okrouhlík et al., 2015). Recently, the higher surface

95 temperature of the ventral body part in a wide gradient of experimental ambient temperatures

96 was confirmed in other species of subterranean rodents from different phylogenetic lineages $(\mathrm{F}$

97 Vejmělka, 2017, unpublished data).

98 The morphology of thermal windows in subterranean mammals is heavily understudied.

99 Šumbera et al. (2007) found notable differences in pelage between the dorsal and ventral body

100 parts in two African mole-rat species. The pelage was four times sparser on the belly than on the

101 back in the giant mole-rat and even nine times sparser in the silvery mole-rat. In the latter

102 species, hairs on the ventral body part were also shorter. Similar differences between ventral and

103 dorsal body regions were found in 15 species of subterranean rodents of different phylogenetic

104 lineages (F Vejmělka, 2015, unpublished data). The importance of fur for heat dissipation has

105 been demonstrated in the South African highveld mole-rat Cryptomys hottentotus pretoriae

106 experimentally, with fur shaving decreasing body temperature, probably as a result of increased

107 heat dissipation (Boyles et al., 2012). This finding indicates that fur characteristics are probably

108 highly relevant for heat dissipation in subterranean species.

109

Skin morphology of the African mole-rats is rather understudied and attention has mainly

110 been paid to the hairless skin of the naked mole-rat (Heterocephalus glaber) (Daly \&

111 Buffenstein, 1998; Sokolov, 1982; Thigpen, 1940; Tucker, 1981). Daly \& Buffenstein (1998)

112 found a dense capillary network in the superficial layers of the dermis in the naked mole-rat $(H$.

113 glaber). Kimani (2013) provided a detailed description of skin morphology of different body 
114 regions in $H$. glaber and the African root-rat Tachyoryctes. He noticed that the skin on the dorsal

115 side is thicker than on the ventral side in both species and explained it by higher resistance to

116 wear during digging. However, while the thickness of skin on the ventral side is comparable in

117 both species, it is much thicker in Tachyoryctes on the dorsal side. This difference in the

118 thickness can be caused by presence of fat in the hypodermis on dorsal side of Tachyoryctes,

119 which can affect thermoregulation.

120 In this study, we focus on skin characteristics of the social F. mechowii, a species in

121 which the role of ventral body size in heat dissipation has been suggested on the grounds of its

122 higher surface temperatures and lower pelage insulation (Šumbera et al., 2007; Okrouhlík et al.,

123 2015). We focus on a comparison of the ventral body part (where higher heat dissipation is

124 expected) with the dorsal body part (where heat dissipation should be limited due to the isolating

125 effect of denser fur). There is a question, of whether heat dissipation on the ventral body part is

126 facilitated due to poor thermal insulation of fur only, or whether the characteristics of the skin

127 also contribute to heat dissipation. If characteristics of the skin on the ventral body part are

128 relevant for heat dissipation, we should expect lower fat content and connectivity allowing heat

129 exchange through gaps in an insulative layer of fat, and also higher vascularization enabling

130 higher heat transport as described in other mammals (Ackermann, 1976; Atlee et al., 1997;

131 Khamas et al., 2012; Mitchell \& Skinner, 2004; Reichard et al., 2010).

132

133 Materials \& Methods

134 Study animals

135 The giant mole-rat Fukomys mechowii is the largest social African mole-rat with a body mass of

136 200-600 g (Kawalika \& Burda, 2007). It is distributed in the Democratic Republic of Congo, 
137 Zambia and Angola, where it inhabits mesic savannas, forests, bushlands, and agricultural fields

138 (Kawalika \& Burda, 2007; Wilson, Lacher \& Mittermeier, 2017). Animals involved in our study

139 were born in captivity and housed at a temperature of $25 \pm 1{ }^{\circ} \mathrm{C}$.

140 We examined skin samples (from the dorsal and ventral sides) of five adult non-breeding

141 females of age 2.5-10.5 years and body weight 186-308 g. The samples were taken with a biopsy

142 needle (6 mm diameter); from two freshly thawed cadavers stored under $-20{ }^{\circ} \mathrm{C}$ and from three

143 perfused specimens (see below). The latter animals were sacrificed in the framework of

144 neuroanatomical studies (cf. Kverková et al. 2018). All five specimens are stored at the Faculty

145 of Sciences, University of South Bohemia, under the accession numbers 2296, 7940, 8280, 9330, 1469653.

147

148 Histology

149 Epidermal and dermal thickness as well as thickness, proportion, connectivity and pattern of

150 dermal fat were assessed in skin samples from all five specimens. From each individual, six skin

151 samples were taken from the dorsal and six from the ventral body part (see Fig. 1 for details of

152 sampling points).

153 Three perfused animals were first processed for analysis of vascularization (see below)

154 and then they were processed by routine histological methods. The two frozen specimens were 155 studied by routine histological methods.

156 Biopsy samples were fixed in $4 \%$ buffered paraformaldehyde (PFA, $\mathrm{pH}=7.2)$, dehydrated

157 by ascending concentrations of ethanol in automatic tissue processor (Leica ASP200S) and

158 embedded in paraffin wax in tissue embedder (Leica EG1150H). Paraffin blocks were sectioned

159 on a rotatory microtome (Leica RM2255) to obtain skin cross-sections, which were then 
160 mounted on a glass slide and stained with hematoxylin and eosin by an autostainer (Leica XL

161 ST5010). The sections were examined under a light microscope (Olympus CX41) with 20×

162 objective magnification and photographed with a digital camera (Olympus DP74).

163 The micrographs were processed using ImageJ 1.48v (Schindelin et al., 2012). A square

164 grid of side length $300 \mu \mathrm{m}$ was randomly overlaid over the entire micrograph. Thickness of

165 epidermis, dermis, and dermal fat was measured on 12 random points and respective mean

166 values were calculated. Thickness of epidermis was measured in the direction perpendicular to

167 its border with dermis. Thickness of dermis and fat layer thickness were measured on the

168 internal-external axis of the animal. Dermis was measured from the epidermal junction to the

169 border of the epimysium (Fig. 2).

170 The extent, connectivity and pattern of the dermal fat were established as follows. A

171 square grid of side length $50 \mu \mathrm{m}$ was overlaid above the section micrograph. The presence of

172 adipocytes within each grid cell throughout the section was determined and used to calculate the

173 extent of dermal fat as a proportion of grid cells with adipocytes present to the total number of

174 grid cells of the section. To evaluate the dermal fat layer connectivity and pattern we defined

175 seven categories of its thickness based on the total thickness along the internal-external axis -0 ,

176 1-50, 51-100, 100-150, 150-200, 200-250, and $>250 \mu \mathrm{m}$. In each section, total width of each

177 thickness category on axis parallel with animal surface was measured and divided by the width

178 of the section to calculate the percentage of the dermal fat thickness categories. We defined fat

179 layer connectivity as a proportion of the width of the section containing fat tissue to the total

180 width of the section and we used the fat layer thickness categories as a measure of adipose tissue 181 pattern. 


\section{Vascularization density}

184 The vessel system of three females was stained with fluorescent dye by transcardial perfusion as 185 described by Li et al. (2008) and studied by confocal microscopy. In short, animals were

186 perfused with $300 \mathrm{ml}$ of heparinized PBS (pH 7.4) followed by perfusion by $120 \mathrm{ml}$ of lipophilic 187 carbocyanine dye (solution of 1,1'-dioctadecyl-3,3,3',3'-tetramethylindocarbocyanine 188 perchlorate). Afterwards, the vascular system was perfused with $300 \mathrm{ml}$ 4\% buffered PFA. On 189 the next day, skin samples were taken by a biopsy needle and stored in $1 \%$ buffered PFA. There 190 were 20 samples per animal in total (see Fig. 1 for details about sampling points). Skin samples 191 were thoroughly rinsed with PBS (four times for 15 min at RT and overnight at $4{ }^{\circ} \mathrm{C}$ ) and transferred to $100 \%$ glycerol through glycerol series $(30 \%, 50 \%, 80 \%$ for 30 min each), mounted on microscope slides and examined under confocal laser scanning microscope (Olympus FV3000) using an objective with 4× magnification (Olympus UPlanSApo4x). Pin-hole size was set automatically to $140 \mu \mathrm{m}$, optical filters were set to match ALEXA 568 (i.e. excitation wavelength $561 \mathrm{~nm}$, emission wavelength $603 \mathrm{~nm}$, detection wavelength 570-620 nm) and grayscale color depth was 12 bit. Other microscope parameters were as follows: laser transmissivity $0.30 \%$, PMT voltage $350 \mathrm{~V}$, gain $1 \times$, offset $2 \%$, sampling speed $12.5 \mu \mathrm{s} /$ pixel and resolution $1024 \times 1024$. Samples were optically sectioned in the Z-axis in 17-20 planes with $30 \mu \mathrm{m}$ distance. Four tiles per plane were imaged and the whole skin sample image in each plane was reconstructed by software stitching (Olympus FV31). To obtain a single plane image with 202 projection of all blood vessels, maximal Z projection of all planes was then performed (ImageJ 203 $1.48 v)$. Contrast of the resulting image was then enhanced using The Curves tool in Gimp GNU

204 GPL v3+ (Solomon, 2009) so that all vessels were clearly visible. For further processing the 205 image color resolution was then reduced to 8-bit. Vessel area and density was then established in 
206 the software AngioTool (Zudaire et al., 2011). Vessel density was calculated as proportion (in $207 \%)$ of vessel area to whole area of the sample.

208 As we did not compare the results statistically, due to the low number of examined 209 individuals, mean values \pm SD are given throughout the text and tables unless stated otherwise. 210

\section{Results}

212 The epidermis of $F$. mechowii consists of 3-10 cell layers covered by a corneous layer. The 213 dermis consists of dense irregular connective tissue with more fibroblasts in the papillary layer 214 and a regular arrangement of hair follicles in the reticular layer (Fig. 2, Fig. 3). The subcutaneous 215 layer (hypodermis) is not clearly delimitated and consists of sparse connective tissue, which is 216 frequently thin and lacks adipocytes. Adipocytes within the dermis are rarely present singularly; 217 they usually form clusters of different sizes and they frequently surrounds growing hair follicles 218 (Fig. 3).

The thickness of the epidermis, dermis and dermal fat layer for the dorsal and ventral skin of each of five specimens are given in Table 1. Mean thickness of the epidermis was higher on the ventral side in most specimens, but the differences between specimens and also between measurements within one side was higher than differences between the dorsal and ventral sides. The difference between mean thickness of the dermis and fat layers on both body sides was negligible, whereas the individual variability was relatively high. The pattern of dermal fat was variable between specimens as well and it did not show any obvious trend (Fig. 4). The relative content of adipose tissue in the dermis was $14.4 \pm 3.7 \%$ on the dorsal and $11.0 \pm 4.0 \%$ on the ventral side (Table 1) and the dermal fat layer connectivity was $98.5 \pm 2.8 \%$ on the dorsal and 
$229100-150 \mu \mathrm{m}$ and the majority of the area was occupied by a fat layer of thickness $50-200 \mu \mathrm{m}$.

230 Detailed proportions of fat layer thickness categories in the dermis of each mole-rat is given in 231 Table S1.

232 Mean vessel density counted as a proportion of the area occupied by vessels inside the 233 maximal projection of whole the explant area (Fig. 6) was $24.6 \pm 3.4 \%(n=3)$ and was slightly 234 higher on the ventral than on the dorsal side, $26.5 \pm 3.3 \%$ and $22.7 \pm 2.3 \%(n=3)$, respectively 235 (Fig. 7). Detailed information about each sample is given in Table S2.

236

237 Discussion

238 We described skin characteristics such as the thickness of epidermis and dermis, the relative 239 extent and pattern of distribution of white adipose tissue in the dermis and the vessel density 240 (vascularization) on the dorsal and ventral sides of the trunk in the strictly subterranean rodent, 241 the giant mole-rat. All these characteristics were expected to play a role in the dissipation of 242 metabolic heat, especially after energy consuming burrowing, which is a typical activity of 243 subterranean mammals. Since we postulate the existence of thermal windows enhancing heat 244 dissipation on the ventral surface of mole-rats, we expected to find morphological differences of 245 the skin between the ventral and dorsal body parts. Surprisingly, we did not find any notable 246 differences between the two body surfaces. Although we studied only a few individuals (note 247 that the evaluation of vessel density requires the sacrifice of living animals), the study still 248 provides good insight into the skin structure and its role in heat dissipation in subterranean 249 rodents, a topic that has not been studied so far. The thickness of epidermis and dermis of the giant mole-rat was within the range found 251 by Sokolov (1982) for different rodent taxa including fossorial ones, i.e. 8-80 $\mu \mathrm{m}$ and 0.2-2.8 
$252 \mathrm{~mm}$ respectively. Sokolov (1982) speculated that scratch diggers have thicker skin on the breast

253 while chisel-tooth diggers have thicker skin on the back, as mechanical protection against wear

254 during digging. Our findings did not confirm this idea, at least for the species in our study, a

255 chisel digger.

256 Dermal adipose tissue is well developed in many mammalian species $;$ and in rodents, it is

257 clearly separated from the subcutaneous fat tissue by panniculus carnosus (Driskell et al., 2014;

258 Wojciechowicz et al., 2013). Although the dermal fat tissue plays a role in protection and

259 regeneration of skin and hair growth cycle (reviewed in Alexander et al., 2015; Guerrero-Juarez

260 \& Plikus, 2018), it also contributes to thermoregulation. The dermal fat layer was found to be

261 thicker in mice facing chronic cold stress and it was calculated that enly a $200 \mu \mathrm{m}$ thick layer of

262 dermal fat reduces heat loss by twofold in mice housed at an ambient temperature of $16^{\circ} \mathrm{C}$

263 below the body temperature (Kasza et al., 2014, 2016). Dermal adipose tissue forms a continuous

264 layer in the laboratory mouse (Kasza et al., 2014) whereas in the naked mole-rat and in the

265 common mole-rat, this tissue consists of either isolated or grouped adipocytes (Daly \&

266 Buffenstein, 1998). In the giant mole-rats, isolated adipocytes are present infrequently, as they

267 form larger clusters situated relatively close to each other, as can be seen from the high

268 connectivity of dermal fat tissue (Fig. 6). The shape of clusters of adipocytes surrounding the

269 hair follicles can be influenced also by the hair cycle as is evident from the Fig. 2 and Fig. 3, and

270 as was described and reviewed in Guerrero-Juarez \& Plikus (2011).

271 Blood vessels play a major role in heat exchange, especially in body parts that facilitate

272 heat dissipation (Bryden and Molyneux, 1978; Tarasoff and Fisher, 1970). Our findings showed

273 that relative vessel density is higher on the ventral than on the dorsal body part, yet the 
274 differences are not so prominent (less than $4 \%$ ) as to cause significant differences in body heat 275 dissipation between both body regions.

276

277

278

279

280

281

282

283

284

285

286

287

288

289

290

291

292

293

294

295

296

In the hitherto studied African mole-rats, no continuous subcutaneous fat layer was found (Daly and Buffenstein, 1998; Sokolov, 1982), while in other mammals, typically, the hypodermis consists mainly of white adipose tissue (Marquart-Elbaz et al., 2001; Matoltsy, 1986;

Scudamore, 2014; Sokolov, 1982). Daly and Buffenstein (1998) as well as Sokolov (1982) found only the aggregations of fat cells within the dermis of the naked mole-rat and common mole-rat, which is in agreement with our findings. Absence of subcutaneous fat tissue and thus the loose skin connection to the deeper fascia allows the integument slidability, which is known from human anatomy of skin in the eyelid or penis (Van De Graaff, 2001). The mobility of loose subcutaneous tissue in subterranean rodents protects skin from injuries (Kawamata et al., 2003), which can facilitate movement underground (Daly \& Buffenstein, 1998). It is known that instead of storing fat in subcutaneous layer, at least some African mole-rats (and probably other subterranean mammals) deposit fat into the intraperitoneal cavity and around the neck (O'Riain, Jarvis \& Faulkes, 1996; Scantlebury, Speakman \& Bennett, 2006). Apart from this, the absence of a subcutaneous fat layer is highly relevant in thermal biology allowing fast dissipation of metabolic heat.

We found no notable differences in the content and connectivity of dermal fat and vascularization density between the ventral body part, i.e. the area with expected heat dissipation function in subterranean rodents (cf. Šumbera et al., 2007; Šumbera, 2019; Cutrera and Antinuchi, 2004) and the dorsal body part. This is in contrast with a prominent difference in the insulative characteristics of fur (hair length and density) between both body areas. In the giant mole-rat, dorsal pelage is four times denser (having the same length), which must certainly 
297 contribute substantially to heat conservation (Šumbera et al., 2007). The important role of fur in

298 mole-rat thermoregulation is indicated also by experiments with fur shaving in Mashona mole-

299 rats (Fukomys darlingi) and highveld mole-rats (Cryptomys hottentotus pretoriae) (Boyles et al.,

300 2012). In mole-rats, heat dissipation could be easily influenced by different patterns of fur

301 characteristics across the body together with some behavioral patterns such as curling up and

302 thus hiding the ventral, less furred area under cold temperatures. Seasonal changes of

303 microenvironmental conditions in burrows could be mitigated by seasonal moulting, which is

304 known in some bathyergids (Hislop \& Buffenstein, 1994).

305

306 Conclusions

307 If we consider all findings of the present study about insulative value of skin and vessel density

308 together with the findings on fur (Šumbera et al., 2007), we may conclude that pelage

309 characteristics are probably the most important factor for dissipation or conservation of body

310 heat in the giant mole-rat. For future studies, a focus on the potential role of hair reduction on

311 the feet or even the short tail on heat dissipation could be interesting. It is known that feet can

312 also eentributenotably, to heat dissipation, so histological analysis of the pads could bring some

313 new information on this issue.

314

315 Acknowledgements

316 We would like to thank Kristina Kverková and Pavel Němec for sharing the three transcardial

317 perfunded specimens and Matěj Lövy for assistance. We also thank to Monika Doll, Katja Haerle 318 and Jutta Mueller for help with processing of samples.

320 References 
321 Ackerman E, 1976. The histogenesis of hair follicles in the zebra and giraffe with special 322 reference to pigmentation and cutaneous vasculature. MSc Dissertation, University of $323 \quad$ Pretoria, pp 104.

324

325

326

327

328

329

330

331

332

333

334

335

336

337

338

339

340

341

Alexander CM, Kasza I, Yen CLE, Reeder SB, Hernando D, Gallo RL, Jahoda CAB, Horsley V, MacDougald OA, 2015. Dermal white adipose tissue: a new component of the thermogenic response. Journal of Lipid Research 56(11): 2061-2069.

Atlee BA, Stannard AA, Fowler ME, Willemse T, Ihrke PJ, Olivry T, 1997. The histology of normal llama skin. Veterinary Dermatology 8: 165-176.

Baldo MB, Antenucci CD, Luna F, 2015. Effect of ambient temperature on evaporative water loss in the subterranean rodent Ctenomys talarum. Journal of Thermal Biology 53: 113-118.

Boyles JG, Verburgt L, McKechnie AE, Bennett NC, 2012. Heterothermy in two mole-rat species subjected to interacting thermoregulatory challenges. Journal of Experimental Zoology 317: 73-82.

Bryden MM, 1964. Insulating capacity of the subcutaneous fat of the southern elephant seal. Nature 203: 1299-1300.

Bryden MM, Molyneux GS, 1978. Arteriovenous anastomoses in the skin of seals. II. The California sea lion Zalophus californianus and the northern fur seal Callorhinus ursinus (Pinnipedia: Otariidae). The Anatomical Record 191: 253-260.

Buffenstein R, 2000. Ecophysiological responses of subterranean rodents to underground habitats. In: Lacey E, Patton JL, Cameron GN (Eds.), Life Underground: The Biology of Subterranean Rodents. The University of Chicago Press, pp 62-110. 
342 Burda H, Šumbera R, Begall S, 2007. Microclimate in burrows of subterranean rodents -

343 revisited. In: Subterranean Rodents: News from Underground. Springer-Verlag, Berlin $344 \quad$ Heidelberg New York, pp 20-33.

345 Cutrera AP, Antenucci D, 2004. Fur changes in the subterranean rodent Ctenomys talarum: 346 Possible thermal compensatory mechanism. Revista Chilena de Historia Natural 77(2): 235$347 \quad 242$.

348 Daly J, Buffenstein R, 1998. Skin morphology and its role in thermoregulation in mole-rats, 349 Heterocephalus glaber and Cryptomys hottentotus. Journal of Anatomy 193: 495-502. 350 Driskell RR, Jahoda CAB, Chuong C-M, Watt FM, Horsley V, 2014. Defining dermal adipose 351 tissue. Experimental Dermatology 23: 629-631.

352 Ebensperger LA, Bozinovic F, 2000. Energetics and burrowing behaviour in the semifossorial 353 degu Octodon degus (Rodentia: Octodontidae). Journal of Zoology 252: 179-186.

354 Feldhamer GA, Drickamer LC, Vessey SH, Merritt JF, Krajewski C, 2015. Mammalogy: 355 Adaptation, Diversity, Ecology. Johns Hopkins University Press, Baltimore, pp 2024. 356 Guerrero-Juarez CF, Plikus MV, 2018. Emerging nonmetabolic functions of skin fat. Nature 357 Reviews Endocrinology 14(3): 163-173.

Hislop MS, Buffenstein R, 1994. Noradrenaline induces nonshivering thermogenesis in both the naked mole-rat (Heterocephalus glaber) and the Damara mole-rat (Cryptomys damarensis) despite very different modes of thermoregulation. Journal of Thermal Biology 19: 25-32. required to maintain intradermal fat and prevent cold stress. PLoS Genet 10(8): e1004514. doi:10.1371/journal.pgen.1004514. 
364 Kasza I, Hernando D, Roldán-Alzate A, Alexander CM, Reeder SB, 2016. Thermogenic

365 profiling using magnetic resonance imaging of dermal and other adipose tissues. JCI Insight $36620161(13):$ e87146.

367 Kawalika M, Burda H, 2007. Giant mole-rats, Fukomys mechowii, 13 Years on the stage. In:

368 Begall S, Burda H, Schleich CE (Eds.) Subterranean Rodents: News from Underground. 369 Springer-Verlag, Berlin Heidelberg New York, pp 205-219.

370 Kawamata S, Ozawa J, Hashimoto M, Kurose T, Shinohara H, 2003. Structure of the rat 371 subcutaneous connective tissue in relation to its sliding mechanism. Archives of Histology $372 \quad$ and Cytology 66(3): 273-279.

373 Khamas WA, Smodlaka H, Leach-Robinson J, Palmer L, 2012. Skin histology and its role in 374 heat dissipation in three pinniped species. Acta Veterinaria Scandinavica 54: 46.

375 Kimani JM, 2013. Comparative skin morphology and wound healing in Kenyan African mole rat 376 (Tachyoryctes ibeanus) and naked mole rat (Heterocephalus glager). Theses, Department of 377 Veterinary Anatomy and Physiology, University of Nairobi, Kenya, pp 155.

378 Klir JJ, Heath JE, 1994. Thermoregulatory responses to thermal stimulation of the preoptic 379 anterior hypothalamus in the red fox (Vulpes vulpes). Comparative Biochemistry and $380 \quad$ Physiology A 109: 557-566.

381 Krattenmacher R, Rübsamen K, 1987. Thermoregulatory significance of non-evaporative heat 382 loss from the tail of the coypu (Myocastor coypus) and the tammar-wallaby (Macropus 383 eugenii). Journal of Thermal Biology 12: 15-18.

384 Kuhn RA, Meyer W, 2009. Infrared thermography of the body surface in the Eurasian otter Lutra $385 \quad$ lutra and the giant otter Pteronura brasiliensis. Aquatic biology 6: 143-152. 
386 Kvadsheim PH, Folkow LP, 1997. Blubber and flipper heat transfer in harp seals. Acta

$387 \quad$ Physiologica Scandinavica 161(3): 385-395.

388 Kverková K, Bělíková T, Olkowicz S, Pavelková Z, O’Riain MJ, Šumbera R, ... \& Němec P, 3892018 . Sociality does not drive the evolution of large brains in eusocial African mole-rats. $390 \quad$ Scientific reports 8(1): 9203.

391 Li Y, Song Y, Zhao L, Gaidosh G, Laties AM, Wen R, 2008. Direct labeling and visualization of 392 blood vessels with lipophilic carbocyanine dye DiI. Nature Protocols 3(11): 1703-1708.

393 Liwanag HEM, Berta A, Costa DP, Budge SM, Williams TM, 2012. Morphological and thermal 394 properties of mammalian insulation: the evolutionary transition to blubber in pinnipeds. 395 Biological Journal of the Linnean Society 107(4): 774-787.

396 Lovegrove BG, 1989. The cost of burrowing by the social mole rats (Bathyergidae) Cryptomys 397 damarensis and Heterocephalus glaber: the role of soil moisture. Physiological Zoology 62: 449-469.

Luna F, Antenucci CD, 2007. Energetics and thermoregulation during digging in the rodent tucotuco (Ctenomys talarum). Comparative Biochemistry and Physiology, Part A 146: 559-564.

401

402 403 404 405 406 407 408
Marquart-Elbaz C, Lipsker D, Sick H, Grosshans R, Cribier B, 2001. Does the "subcutaneous cellular tissue" exist? Annales de Dermatologie et de Venereologie 128(11): 1201-1205.

Martin AL, Irizarry-Rovira AR, Bevier DE, Glickman LG, Glickman NW, Hullinger RL, 2007. Histology of ferret skin: preweaning to adulthood. Veterinary Dermatology 18: 401-411.

Matoltsy AG, 1986. The skin of mammals: Dermis. In: Bereiter-Hahn J, Matoltsy AG, Sylvia Richards K (Eds.) Biology of the integument 2, Vertebrates. Springer, pp 272-277.

McNab BK, 2002. The Physiological Ecology of Vertebrates: A View from Energetics. Cornell University Press, New York, 576.

Peer] reviewing PDF | (2019:11:43267:1:2:NEW 2 Mar 2020) 
409 Mitchell G, Skinner JD, 2004. Giraffe thermoregulation: A review. Transactions of the Royal $410 \quad$ Society of South Africa 59(2): 109-118.

411 Mohler FS, Heath JE, 1988. Comparison of IR thermography and thermocouple measurement of 412 heat loss from rabbit pinna. American Journal of Physiology 254(2): R389-R395.

413 O’Riain MJ, Jarvis JUM, Faulkes CG, 1996. A dispersive morph in the naked molerat. Nature $414 \quad 380: 619-621$.

415 Okrouhlík J, Burda H, Kunc P, Knížková I, Šumbera R, 2015. Surprisingly low risk of 416 overheating during digging in two subterranean rodents. Physiology \& Behavior 138: 236$417 \quad 241$.

418 Reichard JD, Prajapati SI, Austad SN, Keller C, Kunz TH, 2010. Thermal windows on Brazilian 419 free-tailed bats facilitate thermoregulation during prolonged flight. Integrative and 420 Comparative Biology 50: 358-370.

421 Scantlebury M, Speakman JR, Bennett NC, 2006. The energy costs of sexual dimorphism in 422 mole-rats are morphological not behavioural. Proceedings of the Royal Society B-Biological $423 \quad$ Sciences 273: 57-63.

424 Schindelin J, Arganda-Carreras I, Frise E, Kaynig V, Longair M, Pietzsch T, ... \& Tinevez JY, 425 2012. Fiji: an open-source platform for biological-image analysis, Nature methods 9(7): $426 \quad 676-682$.

427 Schmidt-Nielsen K, 1997. Animal Physiology - Adaptation and Environment, $5^{\text {th }}$ edition. 428 Cambridge University Press, pp 611.

429 Scudamore CL, 2014. A Practical Guide to the Histology of the Mouse. Wiley Blackwell, pp 430232. 
431 Sherwood L, Klandorf H, Yancey PH, 2013. Animal Physiology: From Genes to Organisms, $2^{\text {nd }}$ 432 edition. Brooks/Cole, pp 816.

433 Sokolov VE, 1982. Mammal Skin. University of California Press, pp 695.

434 Solomon RW, 2009. Free and open source software for the manipulation of digital images.

435 American Journal of Roentgenology 192(6): W330-W334.

436 Steen I, Steen JB, 1965. Thermoregulatory importance of the beaver's tail. Comparative 437 Biochemistry and Physiology A 15: 267-270.

438 Šumbera R, 2019. Thermal biology of a strictly subterranean mammalian family, the African 439 mole-rats (Bathyergidae, Rodentia) - a review. Journal of Thermal Biology 79: 166-189.

440 Šumbera R, Zelová J, Kunc P, Knížková I, Burda H, 2007. Patterns of surface temperatures in 441 two mole-rats (Bathyergidae) with different social systems as revealed by IR-thermography. $442 \quad$ Physiology \& Behavior 92: 526-532.

443 Tarasoff FJ, Fisher HD, 1970. Anatomy of the hind flippers of two species of seals with 444 reference to thermoregulation. Canadian Journal of Zoology 48(4): 821-829.

445 Thigpen LW, 1940. Histology of the skin of a normally hairless rodent. Journal of Mammalogy $446 \quad 21: 449-456$.

447 Tucker R, 1981. The digging behaviour and skin differentiations in Heterocephalus glaber. $448 \quad$ Journal of Morphology 168:51-71.

449 Van De Graaff KM, 2001. Human Anatomy, Sixth Edition. McGraw-Hill Publishing Company. 450 Vanhoutte G, Verhoye M, Raman E, Roberts M, Van der Linden A, 2002. In-vivo non-invasive 451 study of the thermoregulatory function of the blood vessels in the rat tail using magnetic 452 resonance angiography. NMR in Biomedicine 15: 263-269. 
453 Weissenböck NM, Weiss CM, Schwammer HM, Kratochvil H, 2010. Thermal windows on the 454 body surface of African elephants (Loxodonta africana) studied by infrared thermography. $455 \quad$ Journal of Thermal Biology 35: 182-188.

456 Wilson DE, Lacher Jr. TE, Mittermeier RA, (Eds.), 2017. Handbook of the Mammals of the 457 World - Volume 7. Rodents II Lynx Editions in association with Conservation International $458 \quad$ and IUCN, pp 1008.

459 Withers PC, Cooper CE, Maloney SK, Bozinovic F, Cruz-Neto AP, 2016. Ecological and 460 Environmental Physiology of Mammals. Oxford University Press, USA, pp 590.

461 Wojciechowicz K, Gledhill K, Ambler CA, Manning CB, Jahoda CAB, 2013. Development of 462 the mouse dermal adipose layer occurs independently of subcutaneous adipose tissue and is 463 marked by restricted early expression of FABP4. PLoS One 8(3): e59811. 464 doi:10.1371/journal.pone.0059811.

465 Zelová J, Šumbera R, Okrouhlík J, Burda H, 2010. Cost of digging is determined by intrinsic 466 factors rather than by substrate quality in two subterranean rodent species. Physiology \& 467 Behavior 99: 54-58.

468 Zudaire E, Gambardella L, Kurcz C, Vermeren S, 2011. A computational tool for quantitative 469 analysis of vascular networks. PLoS ONE 6(11): e27385. 


\section{Table $\mathbf{1}$ (on next page)}

Skin characteristics on dorsal and ventral body part in five females of Fukomys mechowii.

Mean \pm SD of epidermis, dermis, and fat tissue thickness and proportion of fat tissue in dermis for dorsal and ventral side of each animal. 


\begin{tabular}{|c|c|c|c|c|c|c|c|c|c|}
\hline \multirow{2}{*}{$\begin{array}{l}\text { Animal } \\
\text { ID }\end{array}$} & \multirow{2}{*}{$\begin{array}{l}\text { Body } \\
\text { mass (g) }\end{array}$} & \multicolumn{2}{|c|}{ Epidermis $(\mu \mathrm{m})$} & \multicolumn{2}{|c|}{ Dermis (mm) } & \multicolumn{2}{|c|}{ Fat layer $(\mathrm{mm})$} & \multicolumn{2}{|c|}{ Fat (\%) } \\
\hline & & Dorsum & Venter & Dorsum & Venter & Dorsum & Venter & Dorsum & Venter \\
\hline 2296 & 187 & $19.4 \pm 6.0$ & $21.6 \pm 7.5$ & $0.7 \pm 0.2$ & $0.9 \pm 0.2$ & $0.1 \pm 0.1$ & $0.2 \pm 0.1$ & $14.3 \pm 2.8$ & $12.1 \pm 3.3$ \\
\hline 7940 & 186 & $20.3 \pm 8.2$ & $19.6 \pm 8.6$ & $1.0 \pm 0.2$ & $1.0 \pm 0.3$ & $0.2 \pm 0.1$ & $0.1 \pm 0.1$ & $10.8 \pm 3.1$ & $8.1 \pm 3.8$ \\
\hline 8280 & 308 & $13.9 \pm 4.6$ & $18.4 \pm 6.3$ & $0.8 \pm 0.2$ & $0.8 \pm 0.3$ & $0.1 \pm 0.0$ & $0.1 \pm 0.1$ & $14.1 \pm 1.8$ & $11.2 \pm 2.5$ \\
\hline 9330 & 301 & $20.9 \pm 6.3$ & $21.4 \pm 6.4$ & $1.2 \pm 0.1$ & $1.3 \pm 0.3$ & $0.3 \pm 0.1$ & $0.2 \pm 0.1$ & $15.0 \pm 3.5$ & $9.6 \pm 1.3$ \\
\hline 9653 & 290 & $10.8 \pm 4.8$ & $14.0 \pm 5.9$ & $0.7 \pm 0.2$ & $0.7 \pm 0.2$ & $0.2 \pm 0.1$ & $0.2 \pm 0.1$ & $17.8 \pm 4.2$ & $14.1 \pm 4.6$ \\
\hline
\end{tabular}




\section{Figure 1}

Schematic location of skin sampling points on the animal.

A - sampling points for evaluation of the area covered by vessels, B - sampling points for histological evaluation of epidermis, dermis and fat tissue characteristics. Samples were taken from both dorsal and ventral body side. 

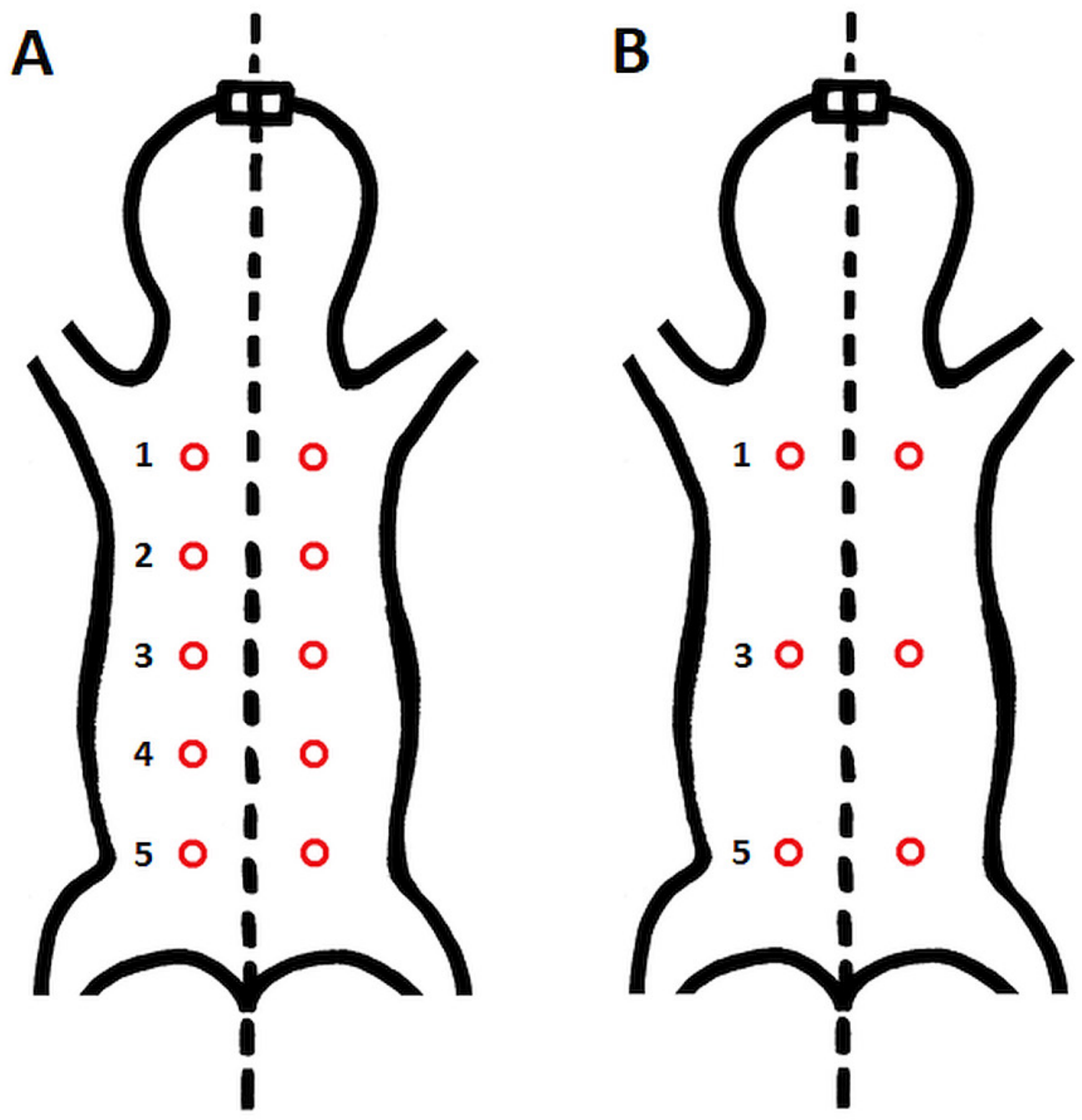
Figure 2

Histological section of skin in Fukomys mechowii.

The section of thickness $4 \mu \mathrm{m}$ was stained by hematoxylin and eosin, D - dermis, E epidermis, $\mathrm{M}$ - muscle layer, $\mathrm{A}$ - adipose tissue. 


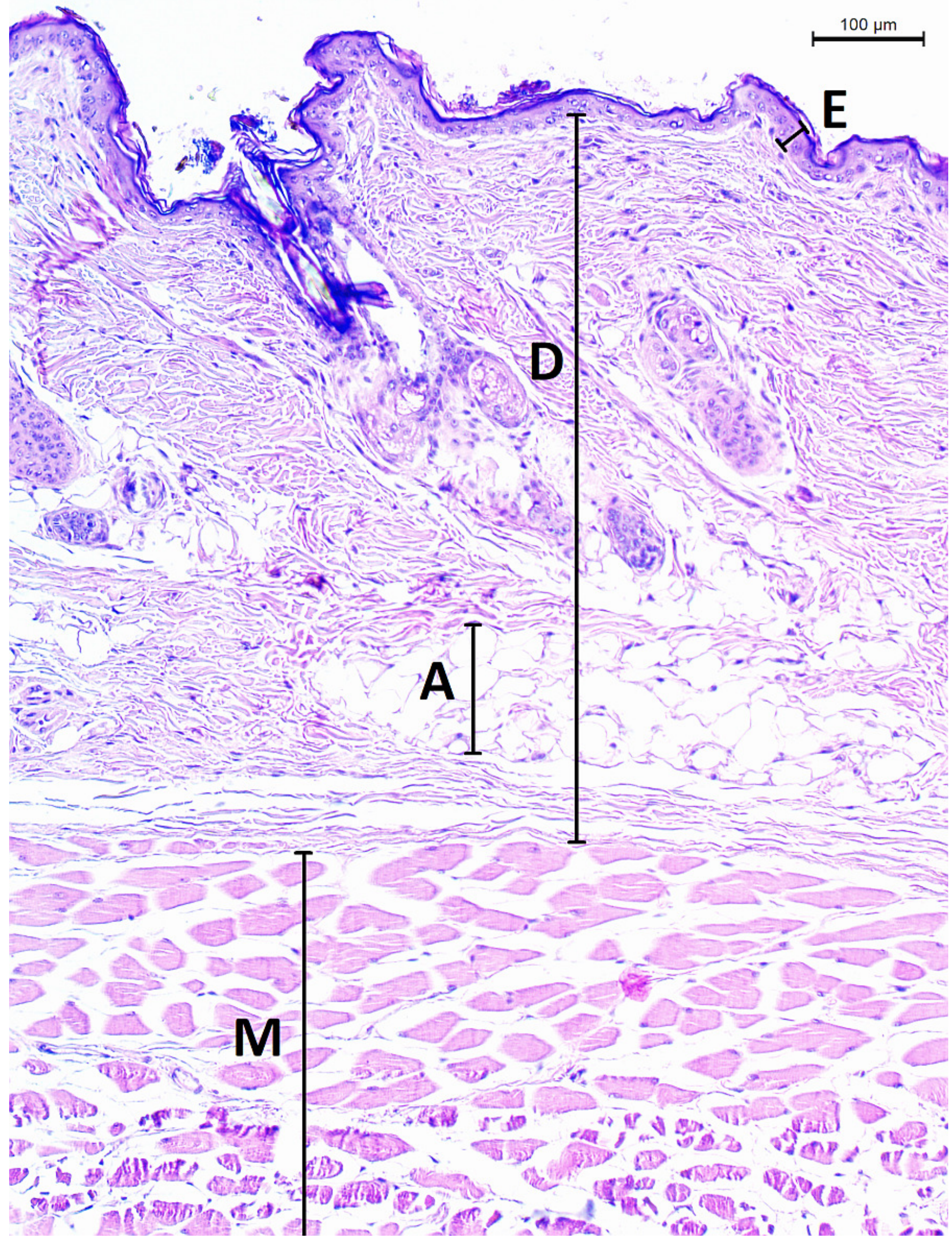


Figure 3

Histological section of skin in Fukomys mechowii showing distribution of adipocytes surrounding hair follicles.

The section of thickness $4 \mu \mathrm{m}$ was stained by hematoxylin and eosin. A - adipocytes, $\mathrm{HF}$ hair follicles with associated glands, $\mathrm{M}$ - muscle. 


\section{$500 \mu \mathrm{m}$}

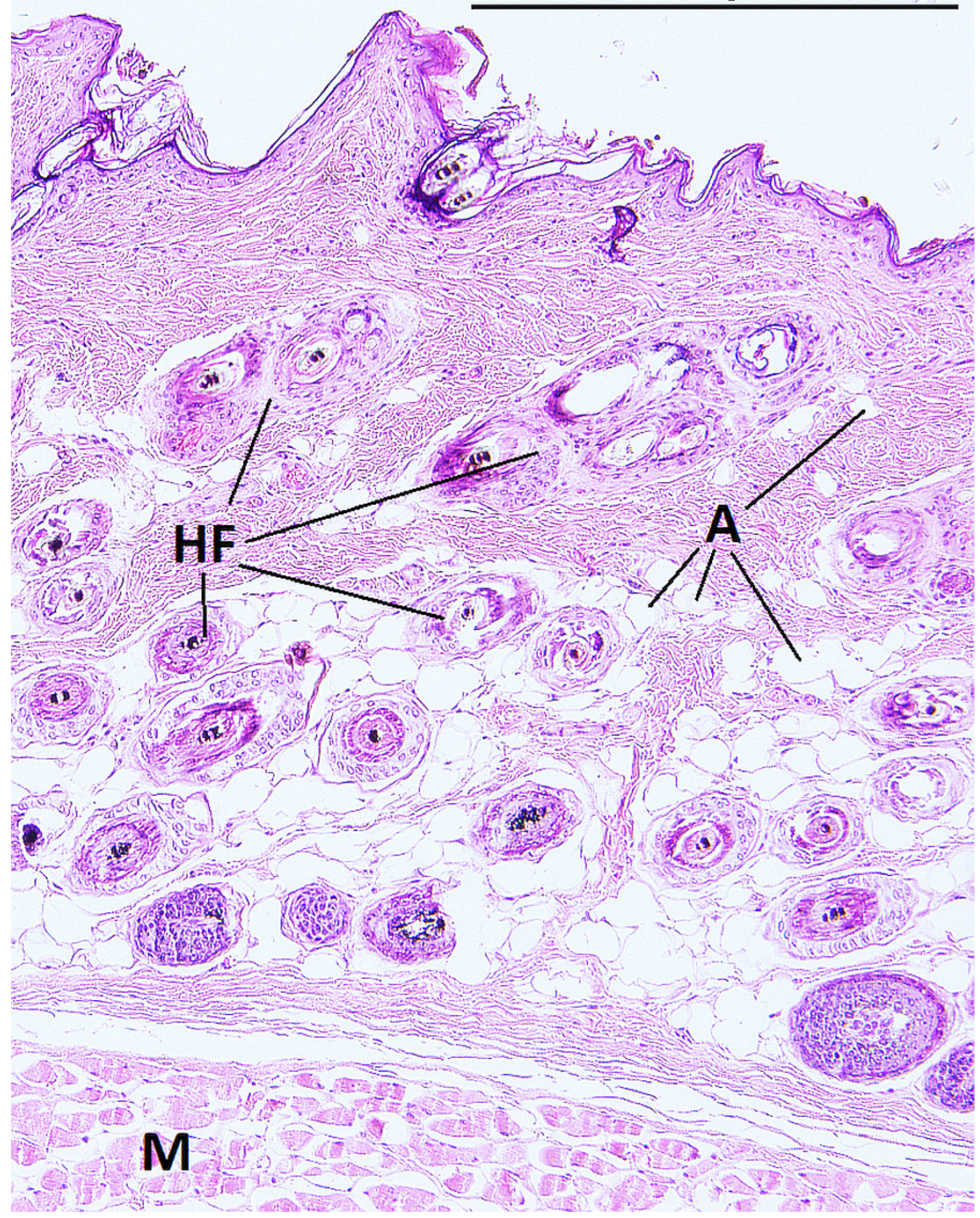


Figure 4

Dermal fat tissue pattern in Fukomys mechowii.

Mean percentage occupied by dermal fat thickness categories in different body parts of each individual: 2296 ( A - anterior, F - middle, K - posterior), 7940 (B - anterior, G - middle, L posterior), 8280 ( $\mathrm{C}$ - anterior, $\mathrm{H}$ - middle, $\mathrm{M}$ - posterior), 9330 ( $\mathrm{D}$ - anterior, I - middle, $\mathrm{N}$ posterior), 9653 ( $\mathrm{E}$ - anterior, J - middle, $\mathrm{O}$ - posterior). Dorsal body part is in black, ventral body part is in grey.

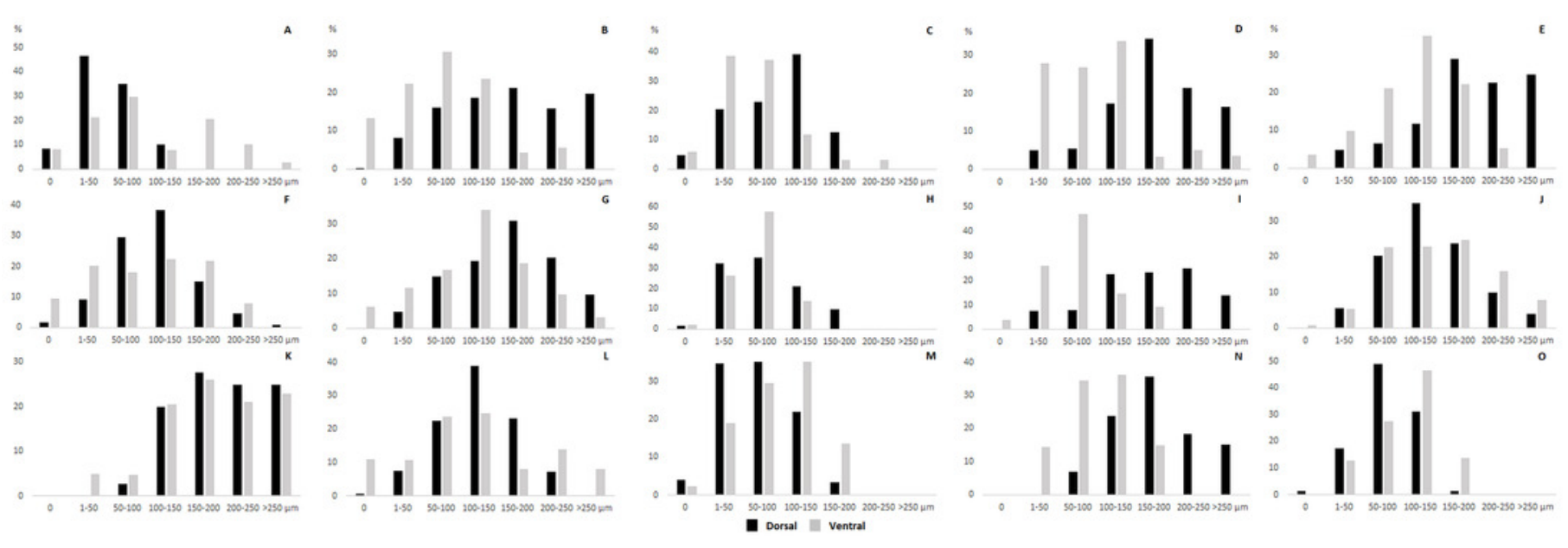


Figure 5

Dermal fat tissue connectivity in Fukomys mechowii.

Proportion of skin sample width containing any dermal fat tissue. Boxplots showing medians (horizontal lines), quartiles (boxes), 5 and 95 percentiles (whiskers) and outliers (black dots) for each animal. Letters A-E denote each specimen (ID numbers 2296, 7940, 8280, 9330, 9653, respectively), D - dorsal and V - ventral body side.
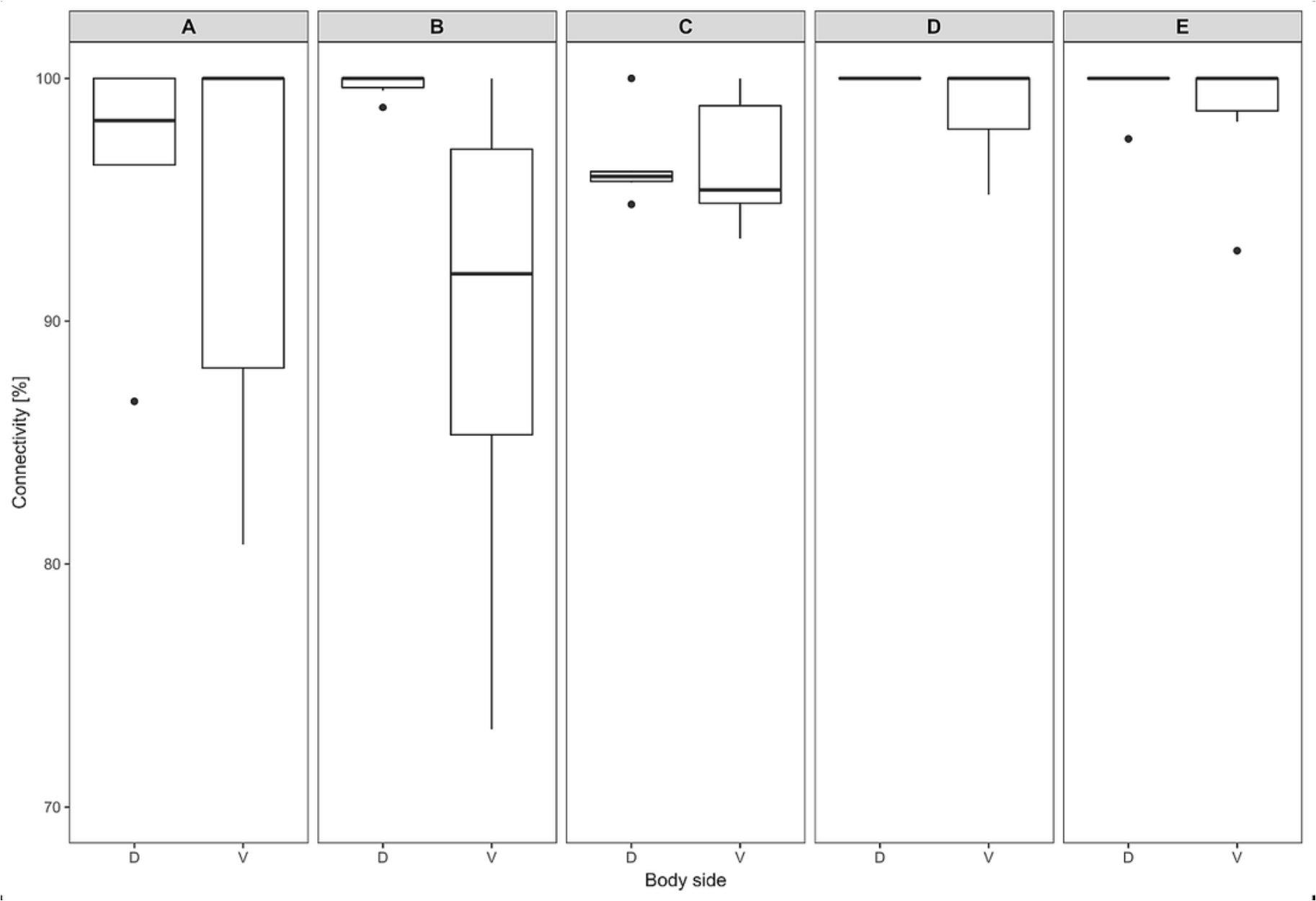
Figure 6

Blood vessels of Fukomys mechowii.

Visualized by Dil labeling, enhanced maximal projection of 17 planes with $30 \mu \mathrm{m}$ distance viewed by confocal microscope. Blood vessels are in white color. 


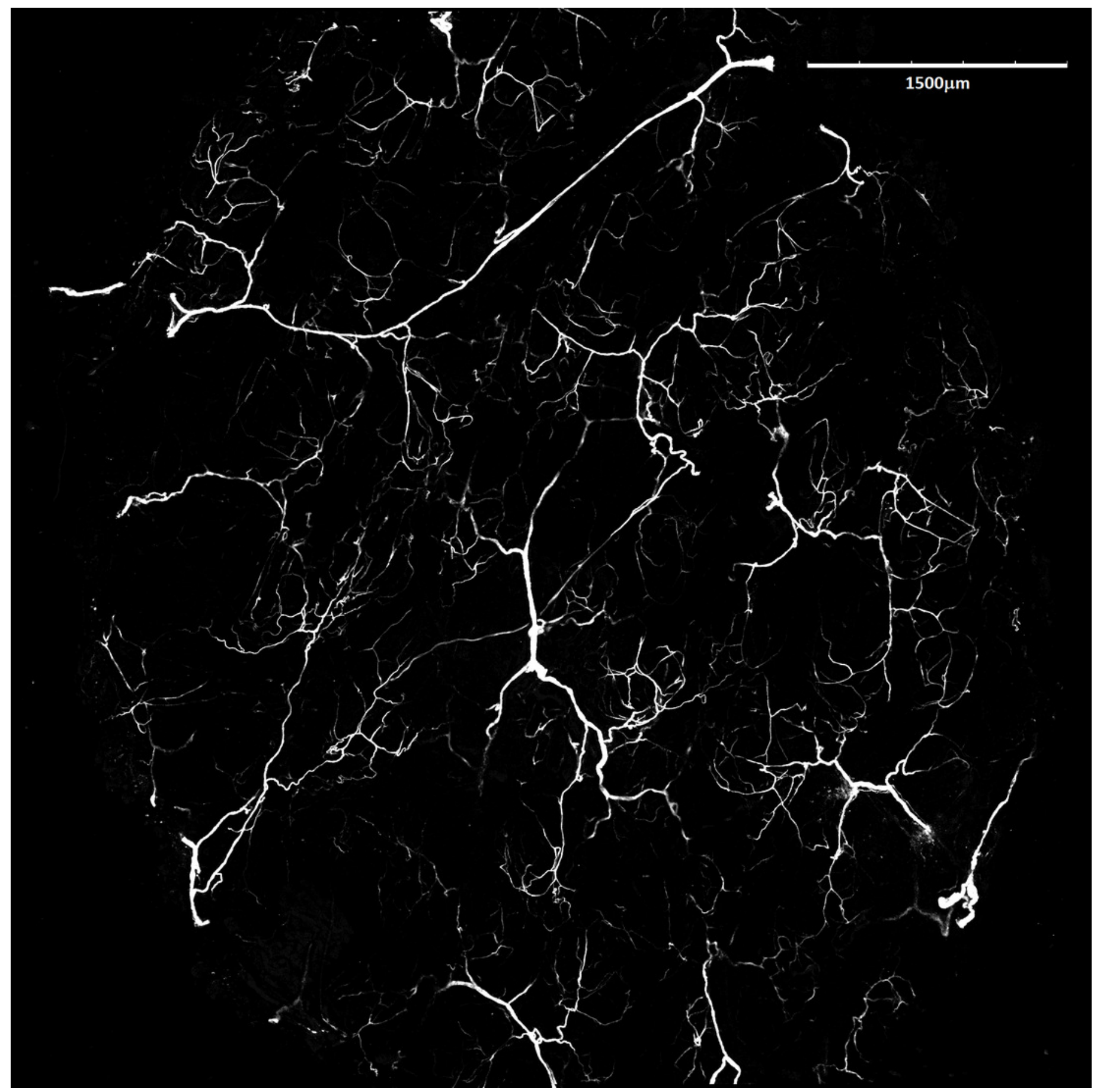


Figure 7

Vascular density in Fukomys mechowii.

Percentage of area covered by vessels in maximal projection of whole skin sample. Boxplots showing medians (horizontal lines), quartiles (boxes), 5 and 95 percentiles (whiskers) and outlier (black dot) in each animal. A, B, C denote specimens 8280, 9330, 9653, respectively, $\mathrm{D}$ - dorsal and $\mathrm{V}$ - ventral body side.

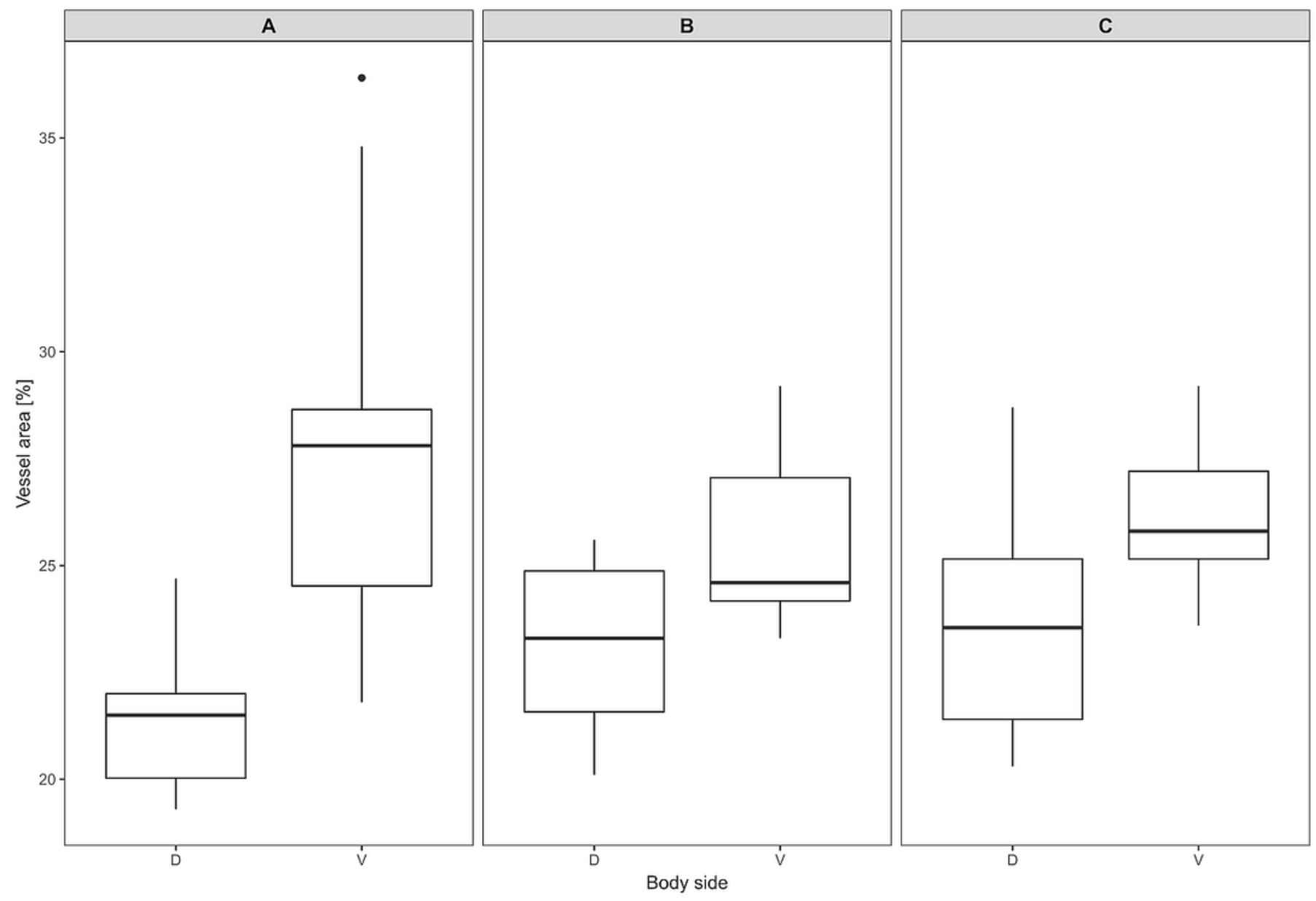

